\title{
Graphene field effect transistor as a radiation and photo detector
}

\author{
Ozhan Koybasi $^{\mathrm{a}}$, Isaac Childres ${ }^{\mathrm{a}}$, Igor Jovanovic ${ }^{\mathrm{b}}$,Yong P. Chen*a,c \\ ${ }^{a}$ Dept. of Physics, Purdue University, West Lafayette, IN 47907 \\ ${ }^{b}$ Dept. of Mechanical and Nuclear Engineering, Penn State University, University Park, PA, 16802 \\ ${ }^{\mathrm{c} B i r c k}$ Nanotechnology Center and School of Electrical and Computer Engineering, Purdue University, \\ West Lafayette, IN 47907
}

\begin{abstract}
We exploit the dependence of the electrical conductivity of graphene on a local electric field, which can be abruptly changed by charge carriers generated by ionizing radiation in an absorber material, to develop novel highperformance radiation sensors for detection of photons and other kinds of ionizing radiation. This new detection concept is implemented by configuring graphene as a field effect transistor (FET) on a radiation-absorbing undoped semiconductor substrate and applying a gate voltage across the sensor to drift charge carriers created by incident photons to the neighborhood of graphene, which gives rise to local electric field perturbations that change graphene resistance. Promising results have been obtained with CVD graphene FETs fabricated on various semiconductor substrates that have different bandgaps and stopping powers to address different application regimes. In particular, graphene FETs made on $\mathrm{SiC}$ have exhibited a $\sim 200 \%$ increase in graphene resistance at a gate voltage of $50 \mathrm{~V}$ when exposed to room light at room temperature. Systematic studies have proven that the observed response is a field effect.
\end{abstract}

Keywords: graphene, transistor, radiation detector, photodetector, field effect

\section{INTRODUCTION}

Graphene [1] has become a focus of rigorous research both in academia and industry due to its many exceptional properties and potential in device applications such as sensors and transistors. The sensitivity of electrical properties of graphene to local electric field changes [2] has led to the idea that graphene configured into a field effect transistor (FET) can be utilized to detect light photons and other types of ionizing radiation, potentially with improved capabilities compared to more conventional radiation detectors, such as high sensitivity and resolution, low electronic noise, low power, and operation at room temperature. The charge carriers induced in the absorber substrate by the incident photons can modify the electric field in the vicinity of graphene, causing a change in the graphene resistivity. The device structure, detection concept and measurement schematics have been presented previously by us and c-workers [3-8] and are depicted in Figure 1. Our prototype graphene FET sensor is made of a graphene layer on an electrically gated undoped radiation absorber substrate with an optional insulating layer in between. A gate voltage, $\mathrm{V}_{\mathrm{G}}$, is applied across the sensor to generate electric field which is varied to find the optimum point on the Dirac curve for a sharp change in graphene resistance. 


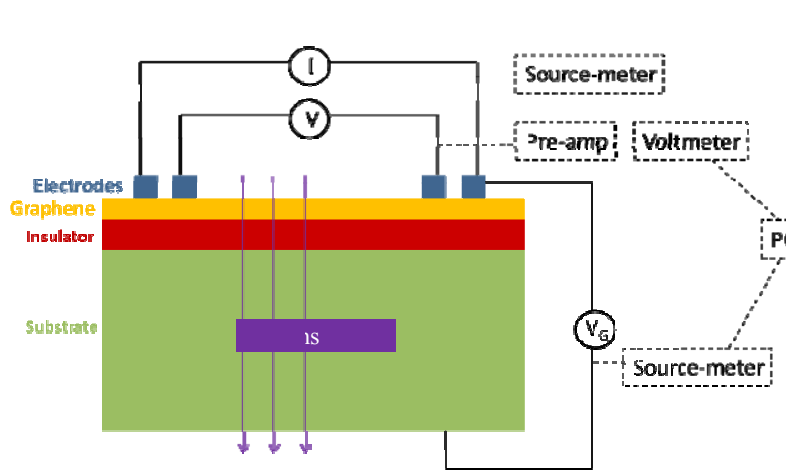

a)

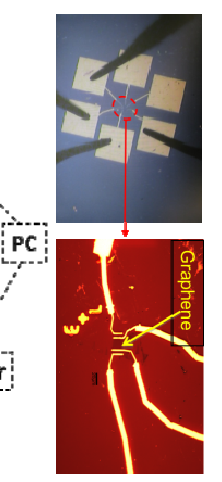

b)

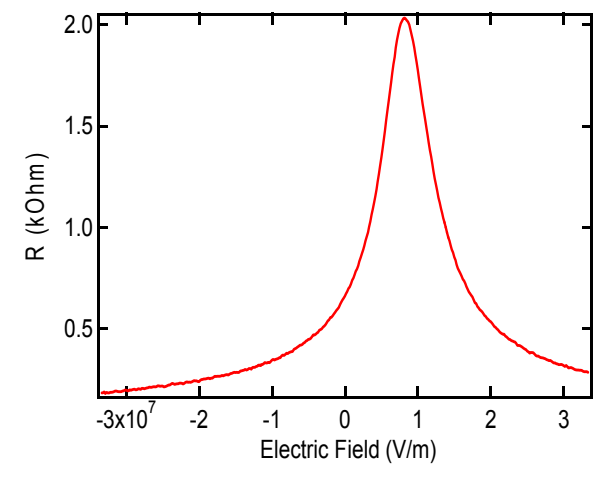

c)

Figure 1. a) Graphene FET (GFET) device structure and experimental schematic for radiation and photo detection. b) 4probe measurement on GFET. Four electrodes are made on graphene in order to allow accurate 4-probe measurement of graphene resistance by eliminating the contact resistance, although a 2-probe measurement could be used in many practical situations. The drain and source electrodes supply the current through the graphene and are used to measure the voltage drop across the graphene. c) Graphene exhibits a sharp peak ("Dirac point") in resistance as a function of the electric field. Data shown are measured in a representative GFET made of exfoliated graphene [2] on a doped Si substrate with $300 \mathrm{~nm}$-thick $\mathrm{SiO}_{2}$ as buffer layer at room temperature.

\section{EXPERIMENTAL RESULTS}

We have fabricated graphene FETs on various semiconductor substrates with different band gaps using CVD graphene [9] to investigate their response to light photons and more energetic photons such as X-rays and gamma rays [3-8], [10]. Although graphene FETs made on (undoped) Si substrate did not exhibit a clear response at room temperature, a significant response was achieved when devices were cooled down to temperatures below $150 \mathrm{~K}$ [10]. Preliminary results showing responses to X-rays and gamma rays have been presented earlier during several IEEE NSS symposia [3-7]. In this paper, we focus on presenting results of detection of light (photodetection).

For room temperature operation, graphene FETs made on a nominally-undoped and higher bandgap substrate SiC $\left(E_{\mathrm{g}}=3.1 \mathrm{eV}\right)$ were studied. Figure 2 shows the graphene resistance as a function of gate voltage for two different measurements; one conducted in dark and the other under a low intensity light source. The plot demonstrates a moderate gate response of graphene FETs, with an increase of graphene resistance for positive gate voltages and decrease for gate biases of the opposite polarity, and how the gate response can be modified by light. Moreover, the relative change of graphene resistance due to light was observed to be approximately proportional to light intensity. Figure 3 indicates the response of the same device to room light at a fixed gate voltage. The measurement was first performed without any gate voltage applied to assure that the device does not show any response to light as expected for the light response described above as due to field effect. At a gate voltage of $50 \mathrm{~V}$, graphene resistance increases by a factor of $\sim 3$ due to light. Although the rise of resistance with the turn on of the light seems quite sharp, it returns very slowly to its original value (before light exposure) when the light is turned off. Due to the gate voltage applied across the device thickness, the charges (holes) generated by the incident light drift to the top surface of the substrate and accumulate in the vicinity of graphene. The slow drop of graphene resistance when the light is turned off is attributed to the lack of a mechanism to drain the charges accumulated underneath graphene. This has been experimentally verified by applying a short voltage pulse with opposite polarity to the gate bias used to operate the 
detector concurrently with the turn off of the radiation source. In this case, graphene resistance has been observed to restore its original value before any light exposure much faster as shown in Figure 3. The dependence of response to light intensity is illustrated in Figure 4. To control the intensity of light on the detector, initially five cleanroom wipers were used between the device and light source, and the number wipers was reduced by one in each measurement interval. Figure 4 clearly shows that graphene resistance increases as the light exposure is increased (as the number of wipers is decreased), and the amount of increase in resistance is proportional to the intensity change.

In addition to $\mathrm{Si}$ and $\mathrm{SiC}$, other semiconductor substrates such as GaAs, CdTe, and CdZnTe have been under investigation to meet needs for different applications. The room temperature operation requires wide bandgap semiconductors such as $\mathrm{SiC}$, while in applications where the main concern is energy resolution, low bandgap materials such as InSb are best suited. On the other hand, detectors made using CdTe and CdZnTe crystals exhibit high energy resolution and detection efficiency (particularly for gamma ray detection), and are usable at room temperature. Although the preliminary results on graphene FETs made on these substrates are encouraging, more detailed studies are needed to evaluate their potential for photon detection.

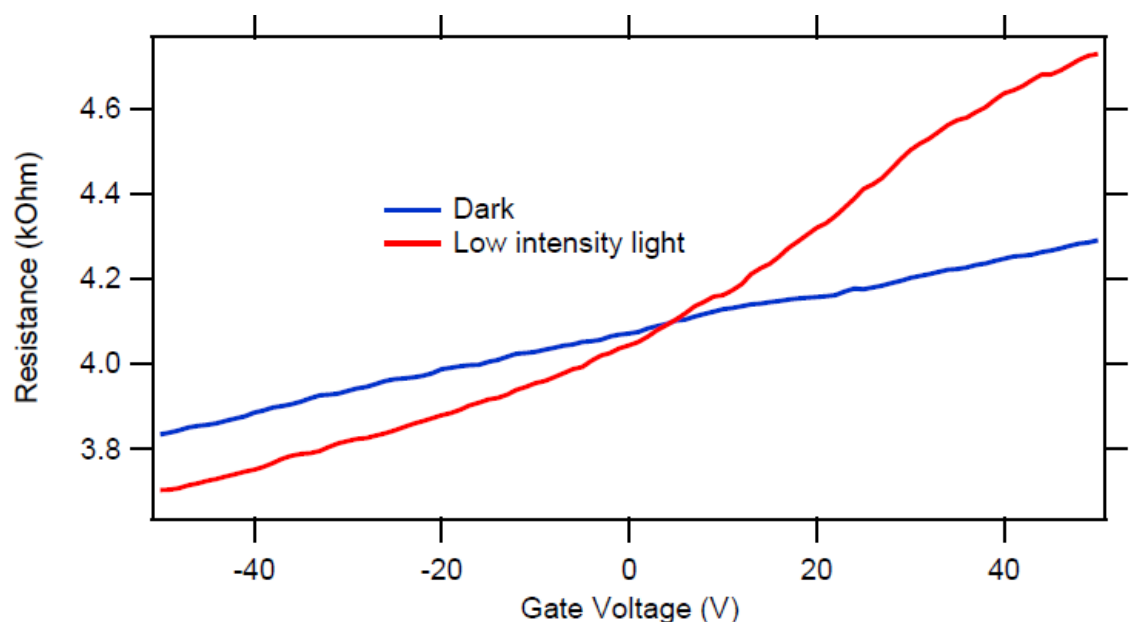

Figure 2. Dirac curves of graphene FET on nominally-undoped SiC in dark and under low intensity light exposure. 


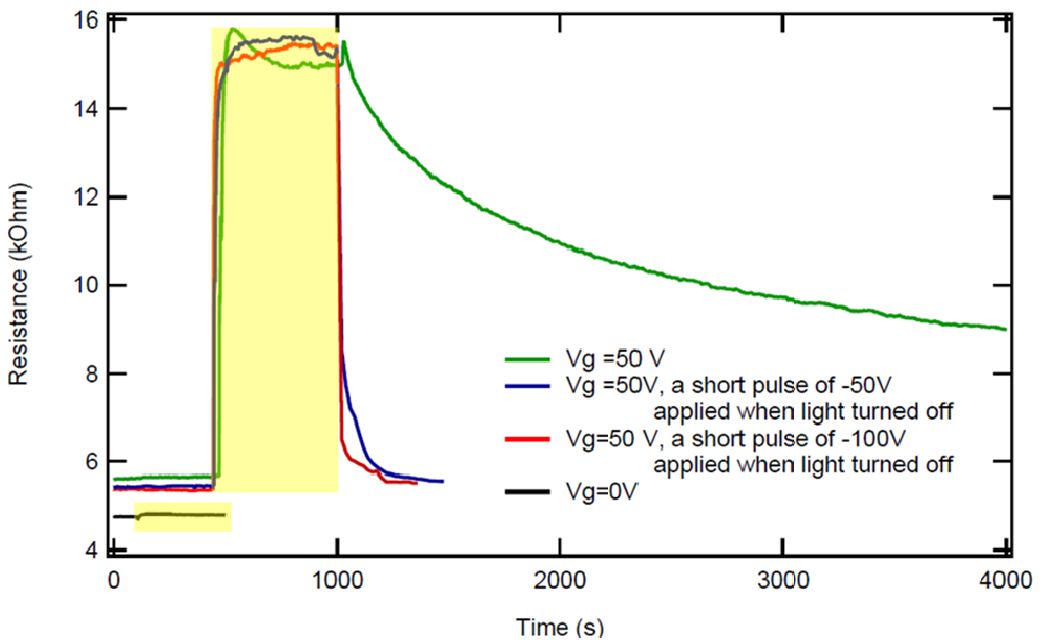

Figure 3. Light response of graphene FET on undoped SiC. Exposure intervals for different curves are shown in yellow boxes. When the turn off of the light is accompanied by a short voltage pulse with opposite sign to the gate voltage applied, resistance returns to its initial value much faster. 


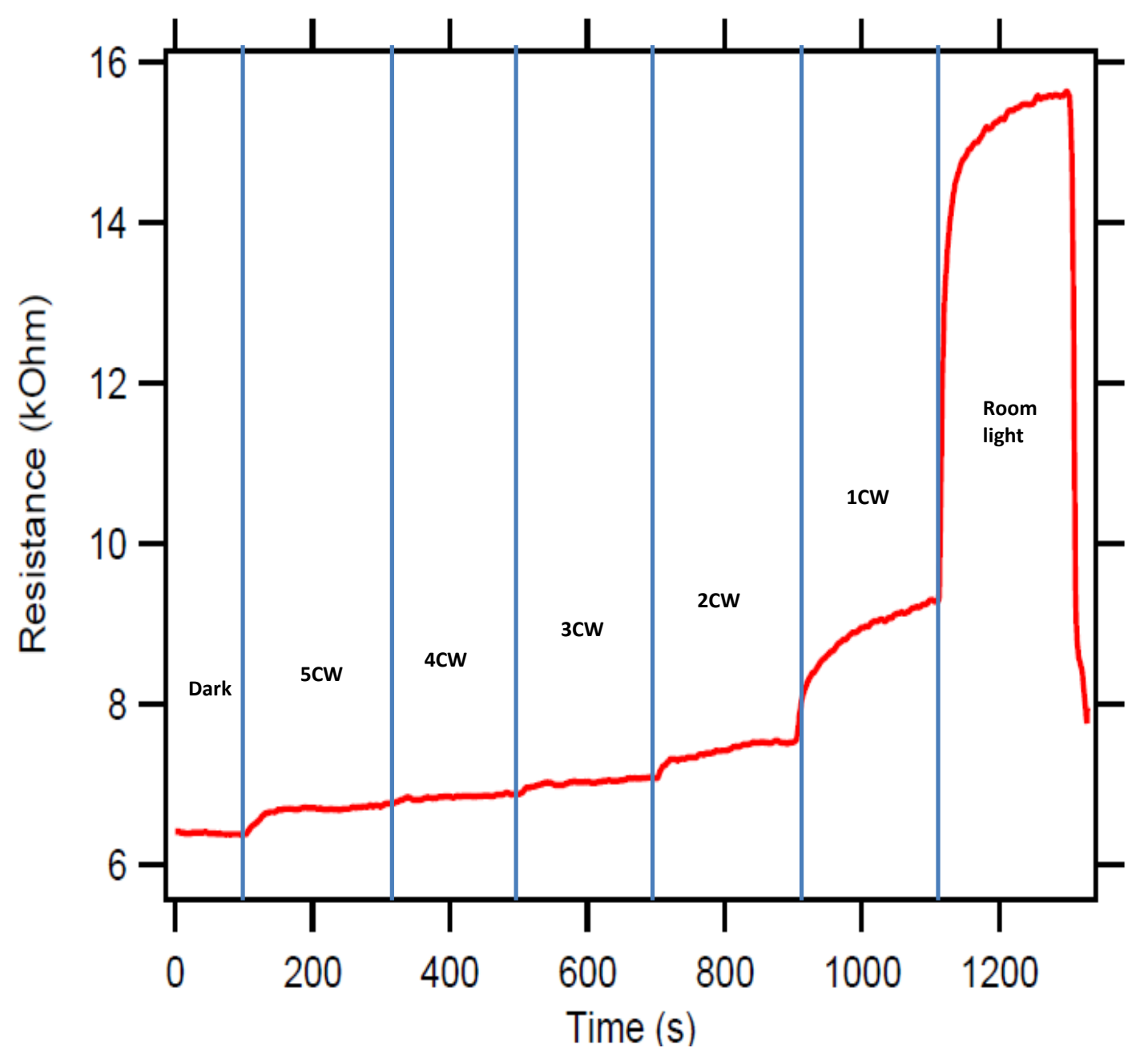

Figure 4. Light response of graphene FET on undoped $\mathrm{SiC}$ as a function light intensity. The number of cleanroom wipers (CW) used to partly block the light are shown in each interval.

\section{DEVELOPMENT OF MORE ADVANCED DEVICE ARCHITECTURES}

As discussed in the previous section, graphene sensors featuring a simple FET structure suffer from low response speed due to the ionized carriers accumulated underneath graphene. We are evaluating more advanced device architectures such DEPFET [11-12] in order to clear the ionized charges from the vicinity of graphene and improve the detection speed of our graphene FET sensors. DEPFET, a detector developed in high energy physics and composed of a field effect transistor incorporated into a fully depleted substrate, provides radiation detection and 
amplification jointly resulting in a very low noise and high resolution. In fact, our graphene sensors exhibit a similar principle of operation to DEPFET in terms of combining detection and amplification, except that, the transistor channel is a p-type inversion layer in case of DEPFET while it is graphene in case of graphene FET. However, our graphene FET has some missing components such as a p-n junction to deplete the substrate, a potential minimum to confine electrons near the transistor channel (graphene), and clear contact to drain the electrons from the potential well after readout. When all these components are incorporated into our graphene FETs, we obtain a DEPFET-like graphene FET structure as depicted in Figure 5, which is expected to resolve the detection speed issue.

We have performed TCAD simulations to fully understand the electrical and charge detection characteristics of DEPFET, and to enhance device performance by improving the design. An n-well is implanted underneath graphene to accumulate the electrons in this region for readout. The electrons are then drained by applying a positive voltage to the "Clear" contact. Figure 6 shows that the ionized electrons accumulated in the n-well disappear in about 5 orders of magnitude shorter time when a positive voltage of $150 \mathrm{~V}$ is applied to the "Clear" contact. All electrons generated in the left side of the detector drift to the n-well, while the majority of the electrons generated on the right side are lost to the "Clear" contact. In order to prevent this loss, a p-well is implanted under the "Clear" contact. The effect of "p-well" is demonstrated in Figure 7. Existence of p-well under the "Clear" contact gives rise to a potential barrier which makes the process of clearing more difficult. In order to control this potential barrier and the potential of the substrate neighboring the internal gate (n-well), a "Clear-gate" electrode is introduced as seen in Figure 5 [13].

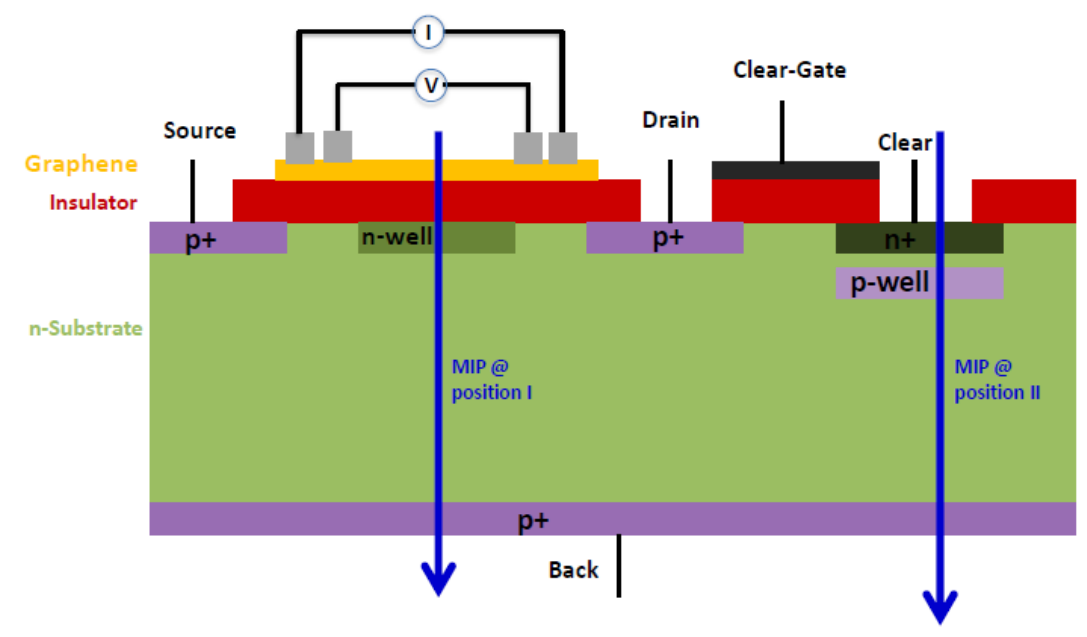

Figure 5. DEPFET-like graphene FET structure. 


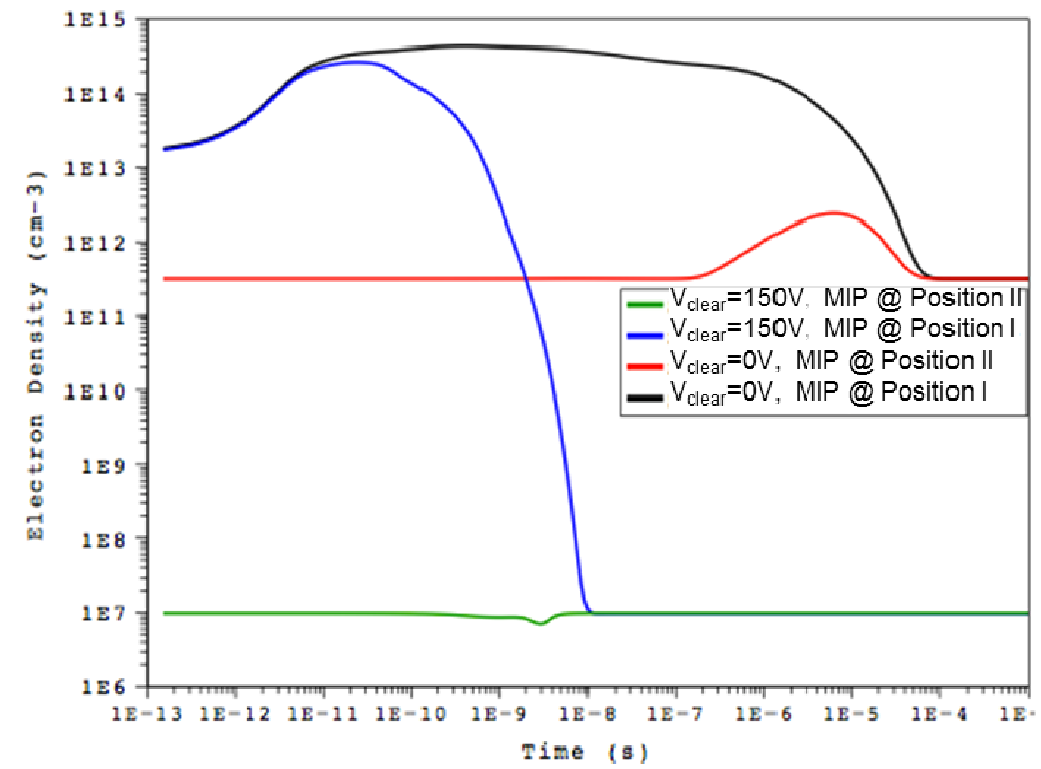

Figure 6. Simulated electron density in n-well vs. time for two different MIP (minimum ionizing particle) positions shown in Figure 5. Both detection and clearing modes are shown. Electrons in n-well disappear in $\sim 5$ orders of magnitude shorter time when positive voltage is applied to "Clear" contact.

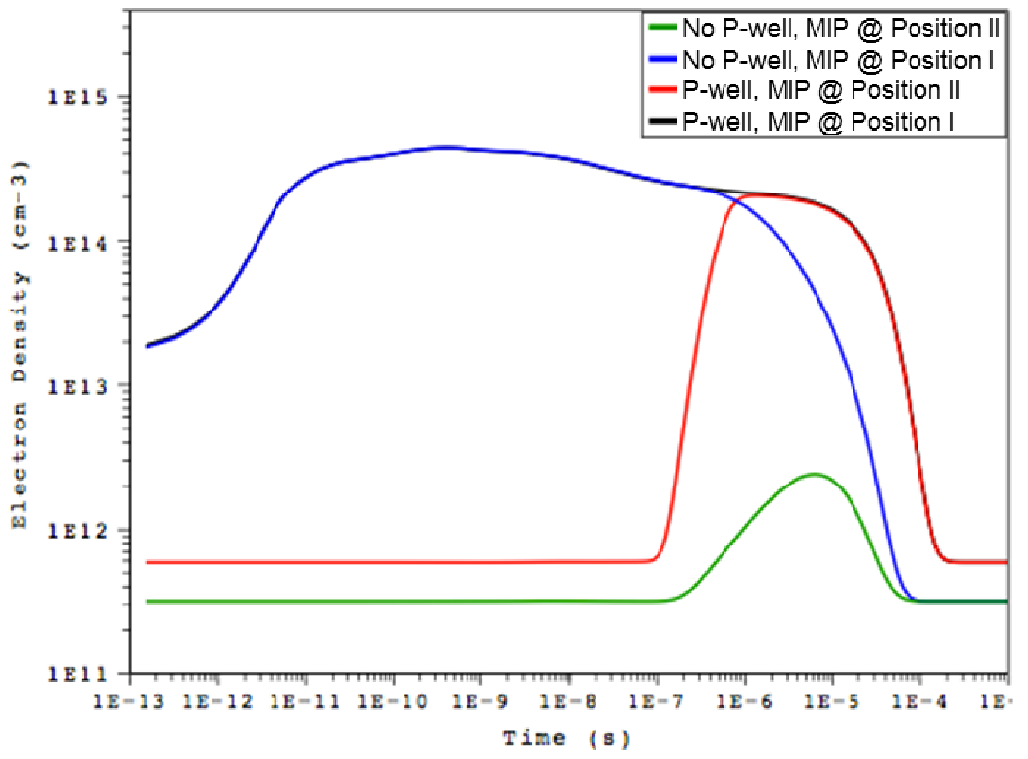

Figure 7. Simulated electron density in n-well vs. time for two different MIP positions shown in Figure 5 with and without p-well under "Clear" electrode. 


\section{CONCLUSION}

Strong field effect response of gated CVD graphene FETs to photons at room temperature strengthens their potential use as a high performance photodetector with a novel detection concept. The speed performance is aimed to be significantly enhanced by configuring graphene into a DEPFET architecture instead of the current simple FET structure. TCAD simulations have provided us with insights to realize high speed graphene on DEPFET devices.

\section{ACKNOWLEDGMENT}

This work has been funded by Department of Homeland Security under award 2009-DN-077-ARI036- 02.

\section{REFERENCES}

[1] A.K. Geim and K.S. Novoselov., "The Rise of Graphene" Nature Materials, 6, 183 (2007).

[2] K.S. Novoselov, et al. "Electric field effect in atomically thin carbon films", Science 306, 666 (2004).

[3] Mike Foxe, Gabriel Lopez, Isaac Childres, Romaneh Jalilian, Caleb Roecker, John Boguski, Igor Jovanovic and Yong P. Chen, "Detection of Ionizing Radiation Using Graphene Field Effect Transistors", IEEE Nuclear Science Symposium (NSS) Conference Records, p.90, Orlando, FL (2009)

[4] Amol Patil, Gabriel Lopez, Michael Foxe, Isaac Childres, Caleb Roecker, John Boguski, Igor Jovanovic, and Yong P. Chen, "Graphene Field Effect Transistors for Detection of Ionizing Radiation", IEEE Nuclear Science Symposium (NSS) Conference Record, p.674, (2010)

[5] Michael Foxe, Caleb Roecker, John Boguski, Isaac Childres, Gabriel Lopez, Amol Patil, Yong P. Chen, and Igor Jovanovic, "Numerical Model of Graphene-Based Radiation Detector Response", IEEE Nuclear Science Symposium (NSS) Conference Record, p.667, (2010)

[6] A. Patil, O. Koybasi, G. Lopez, M. Foxe, I. Childres, C. Roecker, J.Boguski, J. Gu, M. L. Bolen, M. A. Capano, P. Ye, I. Jovanovic, and Y. P. Chen, "Graphene Field Effect Transistoras Radiation Sensor", IEEE NSS-MIC Conference Record (2011)

[7] Michael Foxe, E. Cazalas, H. Lamm, A. Majcher, C. Piotrowski, Isaac Childres, Amol Patil, Yong P. Chen, Igor Jovanovi, "Graphene based neutron detectors", IEEE NSS-MIC Conference Record (2011)

[8] Mike Foxe, Gabriel Lopez, Isaac Childres, Romaneh Jalilian, Caleb Roecker, John Boguski, Igor Jovanovic and Yong P. Chen, "Detection of Ionizing Radiation Using Graphene Field Effect Transistors", accepted by IEEE Transactions on Nanotechnology (2012)

[9] Helin Cao, Qingkai Yu, Luis Jauregui, Jifa Tian, Wei Wu, Zhihong Liu, Romaneh Jalilian, Daniel K. Benjamin, Zhigang Jiang, Jiming Bao, Steven S. Pei and Yong P. Chen, "Electronic Transport in Chemical Vapor Deposited Graphene Synthesized on Cu: Quantum Hall Effect and Weak Localization", Applied Physics Letters 96, $122106(2010)$

[10] G. A. Lopez, "Graphene Field Effect Transistors for Applications in Radiation Detection," M.S. thesis, School of Electrical and Computer Engineering, Purdue Univ., West Lafayette, IN, 2010.

[11] J. Kemmer et al., "Experimental confirmation of a new semiconductor detector principle", Nuclear Instruments and Methods in Physics Research A 288, 92 (1990).

[12] G. Lutz et al., "DEPFET-detectors: new developments" Nuclear Instruments and Methods in Physics Research A 572, 311 (2007).

[13] C. Sandow et. al., "Clear-performance of linear DEPFET devices" Nuclear Instruments and Methods in Physics Research A 568, 176 (2006). 